\title{
Hemodynamic Changes in the Brachial Artery Induced by Acupuncture Stimulation on the Lower Limbs: A Single-Blind Randomized Controlled Trial
}

\author{
Masashi Watanabe, Shin Takayama, Atsushi Hirano, Takashi Seki, and Nobuo Yaegashi \\ Department of Traditional Asian Medicine, Graduate School of Medicine, Tohoku University, Sendai 980-8575, Japan \\ Correspondence should be addressed to Takashi Seki, tseki.tohoku@gmail.com
}

Received 9 August 2012; Revised 16 October 2012; Accepted 30 October 2012

Academic Editor: Wolfgang Schwarz

Copyright (c) 2012 Masashi Watanabe et al. This is an open access article distributed under the Creative Commons Attribution License, which permits unrestricted use, distribution, and reproduction in any medium, provided the original work is properly cited.

Acupuncture is commonly performed at acupoints. No comparisons of quantitative physiological alterations in the brachial artery (BA) induced by the stimulation of different acupoints in the lower limbs have been performed in humans. Therefore, we investigated changes in blood flow volume (BFV) in the BA as an indicator of the physiological effects induced by stimulation at 3 points. Seventy-five healthy participants aged $33 \pm 9$ years (mean \pm SD) were enrolled and randomly assigned to 3 groups; they received stimulation at 3 different points located on the lower limbs: ST36, LR3, and a non-acupoint. Stimulation was performed bilaterally with manual rotation of the needles. Using ultrasonography, BFV was measured continuously from rest to 180 seconds after stimulation. LR3 stimulation significantly increased BFV compared to that before needle insertion. Meanwhile, stimulation at ST36 and the non-acupoint significantly decreased BFV compared to that before needle insertion. Stimulation at LR3 elicited a significant increase in BFV compared to that at ST36 and the non-acupoint. The results suggest that the stimulation of different points on the lower limbs causes distinct physiological effects on BFV in the BA.

\section{Introduction}

Acupuncture is an important facet of traditional Chinese medicine [1]. Acupuncture points, called acupoints, are gateways located on meridians that can restore the flow of $q i[2]$. The treatment of specific acupuncture points depends on the particular diagnosis [3]. The acupuncture theory is based on the meridian system, which connects acupoints to the organs. However, it has been difficult to verify the meridian and acupoint structures as well as and the connections between organs and meridians. The Zusanli (ST36) acupoint is located on the stomach meridian. This acupoint is known to be effective for the treatment of digestive system disorders; it improves digestive function and decreases abdominal pain $[1,4]$. The Taichong (LR3) acupoint is located on the liver meridian; stimulation at this point is known to regulate liver function $[1,4]$. One of the functions of the liver is to regulate the free movement of qi. Stagnation of liver qi may impede blood circulation [4] and cause frequent limb chills.
However, there is currently no evidence supporting the findings mentioned above.

Blood flow volume (BFV) is an important index for representing the condition of organs and tissues. Therefore, we employed BFV as a quantitative indicator of the effects of traditional interventions on the human body. There are some reports in the literature about the hemodynamic responses to acupuncture stimulation at several acupoints [5-9]. Acupuncture is reported to be effective for treating Raynaud syndrome [10] and chills in the hand [11] as well as improving blood circulation in the forearm. However, these reports only show changes in temperature or blood flow at the skin surface. We previously reported the effects of acupuncture at a single acupoint on the BFV of the upper limb in humans, measured by ultrasonography $[12,13]$. In addition, we reported the effects of acupuncture on BFV in the superior mesenteric artery by acupuncture at different acupoints in the lower limbs [14]. To our knowledge, no study has reported changes in the BFV of the brachial 
artery (BA) induced by acupuncture stimulation at different acupoints on the lower limbs. Therefore, this study aimed to clarify the effects of acupuncture stimulation at 2 different acupoints, ST36 and LR3, and a non-acupoint on the hemodynamics in the BA of healthy participants.

\section{Methods}

2.1. Participants. Seventy-five healthy adult volunteers $(45$ men and 30 women) aged $33 \pm 9$ years (mean \pm SD; range 20-53 years) were enrolled in this study. No participants had cardiovascular disease. None of the participants took any medicine 1 month before the experiment. All participants were examined after fasting and abstaining from alcohol and caffeine for at least 3 hours. The study protocol was approved by the Ethics Committee of Tohoku University, Graduate School of Medicine. All participants provided written informed consent prior to the beginning of the experiment.

2.2. Setting. The participants rested in the supine position in a quiet, air-conditioned room (temperature $25-26^{\circ} \mathrm{C}$ ). Three monitoring electrocardiographic electrodes were attached to the anterior part of the chest of each participant. Four electrodes for impedance cardiography (ICG) (Bioz ICG Module, Dash 3000囚, GE Healthcare, USA) were placed at the base of the neck and the level of the xiphoid process in the midaxillary line. ICG utilizes 4 dual sensors on the neck and chest to apply low-amplitude high-frequency alternating electrical current to the participant's thorax. Pulsatile changes in BFV and velocity are measured as changes in impedance. These changes are subsequently synchronized with the electrocardiogram to automatically calculate hemodynamic parameters such as stroke volume and the cardiac index (CI) [15]. ICG is a noninvasive monitoring method that allows the measurement of the CI according to the changes in thoracic resistance that result from variations in intrathoracic BFV $[16,17]$. The CI was calculated on the basis of the stroke volume, heart rate, and body surface area measured by ICG [18]. The systemic vascular resistance index (SVRI) was calculated using the CI and blood pressure. Blood pressure was measured with an oscillometer (BP-608 Evolution IIR, Colin Healthcare Co. Ltd., Kyoto, Japan) on the left upper arm. BA hemodynamics was measured by an ultrasonograph (Prosound $\alpha 10 \mathrm{~B}$, Hitachi-Aloka Medical Ltd., Tokyo, Japan) on the right arm; this system contains a high-resolution linear array transducer $(13 \mathrm{MHz})$ and

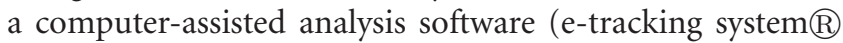
Hitachi-Aloka Medical Ltd., Tokyo, Japan). The software automatically detected the vessel edge and measured the vessel diameter and BFV continuously [19]. The combination of ultrasonography and pulsed Doppler enables the noninvasive investigation of the blood flow in small vessels such as the coronary, splenic, and adrenal arteries as well as the superior mesenteric artery and BA [20]. The right arm was fixed, and the right BA was scanned longitudinally where the vessel diameter and Doppler wave readings were stable. The transducer was fixed in a special probe holder
(MP-PH0001ß, Hitachi-Aloka Medical Ltd., Tokyo, Japan) at the site where the clearest B-mode image of the anterior and posterior vessel wall was obtained (Figure 1). Care was taken to avoid compressing the artery. The BA diameter was monitored automatically when the tracking gate was placed on the intima of the vessel. The waveform of the changes of vessel diameter over the cardiac cycle was displayed in

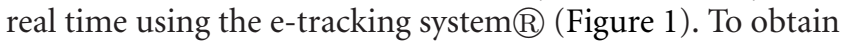
accurate measurements, the Doppler angle was maintained at $60^{\circ}$ or less $[21,22]$. BFV was calculated automatically as the Doppler flow velocity (corrected for the angle) multiplied by heart rate and vessel cross-sectional area [21-23]. To ensure consistent images were obtained, the probe was maintained in the same position throughout the test using a special holder. The e-tracking system $\mathbb{B}$ automatically measured changes of vessel diameter with a precision of $0.01 \mathrm{~mm}$. The use of this system avoids operator bias, increases reproducibility, and improves accuracy. The system and software were developed to measure flowmediated dilatation (FMD), which is usually measured at the BA $[24,25]$. Ultrasonography is a noninvasive method for evaluating blood flow velocity. Blood flow changes rapidly in the arteries of the extremities, especially in the peripheral arteries [26]. Changes in venous return due to respiration cause oscillations in stroke volume and blood pressure [27]. Thus, the arterial pulse should be modified by breathing [28]. Therefore, the participants were asked to breathe every 6 seconds during the test, and hemodynamic parameters were calculated as the average values of each 6-second period to minimize the influence of respiration. The following hemodynamic parameters were determined: (1) blood pressure ( $\mathrm{mmHg}$ ), (2) heart rate (beats/min), (3) cardiac index $\left(\mathrm{L} \cdot \mathrm{min}^{-1} \cdot \mathrm{m}^{-2}\right)$, and (4) blood flow volume $\left(\mathrm{mL} \cdot \mathrm{min}^{-1} \cdot \mathrm{m}^{-2}\right)$.

2.3. Study Protocol. A single-blind randomized controlled trial was performed. The experimental outline of the study is shown in Figure 2 . All participants $(n=75)$ were randomly assigned to 1 of 3 groups receiving the following treatments: (1) needle stimulation at ST36 $(n=25$, Zusanli: located on the lower leg, 3 units below the lateral "eye" of the knee, approximately 1 finger width lateral to the tibia [29]) (Figure 3); (2) needle stimulation at LR3 ( $n=25$, Taichong: located on the foot, 1.5-2 units above the web between the first and second toes [29]) (Figure 3); (3) needle stimulation at a non-acupoint $(n=25$ : located on the lower leg, 3 units lateral to and below ST36, midpoint of the stomach and gallbladder meridian) (Figure 3). The participants had no knowledge about acupuncture or acupoints.

After the ultrasonograph was positioned, the participants rested in the supine position for 10 minutes. We then measured the BA hemodynamics, blood pressure, heart rate, and the $\mathrm{CI}$ at rest (i.e., before needle insertion), during needle stimulation, and 30, 60, and 180 seconds after needle stimulation. Needle stimulation was performed by a licensed acupuncturist. Disposable fine stainless-steel needles $(0.16 \mathrm{~mm}$ diameter, $40 \mathrm{~mm}$ length, Seirin Co. Ltd., Shizuoka, Japan) were inserted bilaterally on the target point 


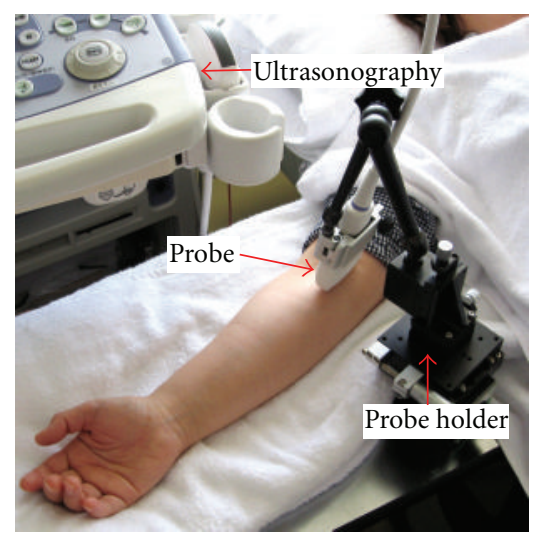

(a)

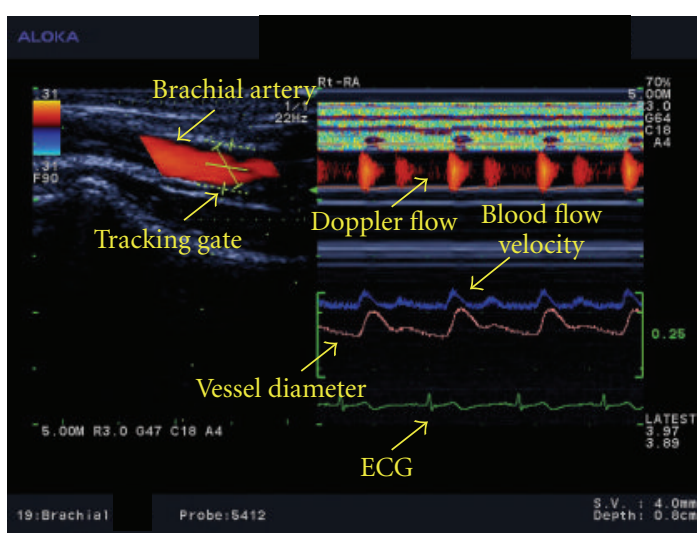

(b)

FIGURE 1: (a) An ultrasonographic measurement of the brachial artery with a special probe holder (MP-PH0001ß), Hitachi-Aloka Medical Ltd., Tokyo, Japan). (b) Hemodynamic data obtained by ultrasonography. The left image shows the vessel image and position of the tracking gate of the brachial artery. The right image shows the changes in vessel diameter, Doppler flow, and blood flow velocity determined by an automated edge detection device and computer analysis software (e-tracking system®, Hitachi-Aloka Medical Ltd., Tokyo, Japan).

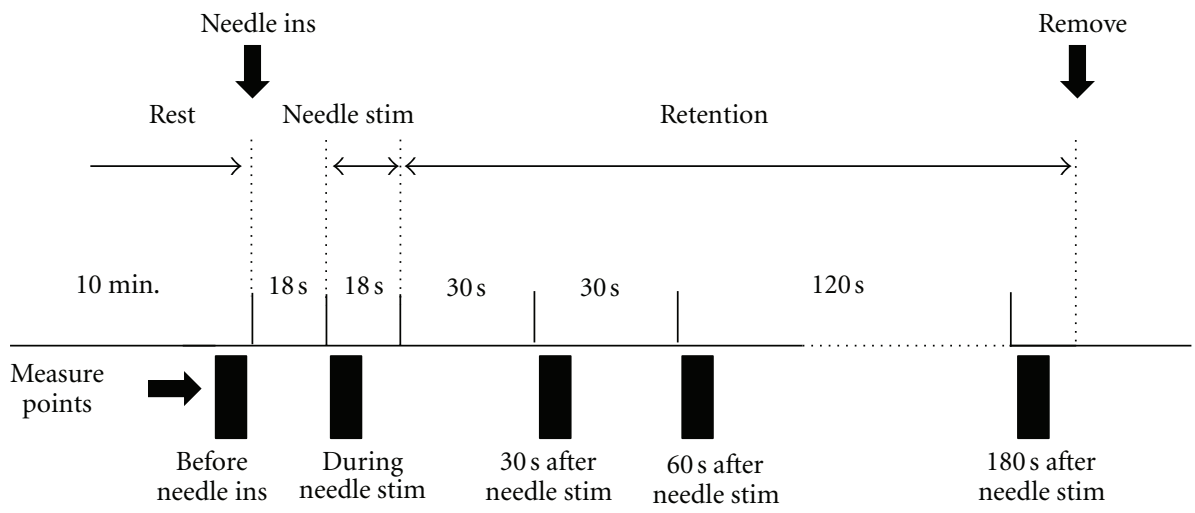

FIGURE 2: Experimental diagram. Before needle insertion (ins) and stimulation (stim) bilaterally at ST36, LR3, and the non-acupoint. After the needles were inserted, needle stimulation was applied for $18 \mathrm{~s}$ with manual rotation. The needles were retained for $180 \mathrm{~s}$ after needle stimulation and then removed. Hemodynamic parameters were measured before needle insertion, during, and 30, 60, and $180 \mathrm{~s}$ after needle stimulation. min.: minutes, s: seconds.

and maintained at a depth of $10 \mathrm{~mm}$ during the test to ensure they reached the muscle. After the needles were inserted, the stimulation was performed for 18 seconds by rotating the needles $<90^{\circ}$ manually. The needles were retained for the duration of the test following stimulation and were then removed.

2.4. Statistical Analysis. Statistical analysis was performed with PASW software (version 17.0, SPSS Japan Inc., Tokyo, Japan). Comparisons between the ST36, LR3, and nonacupoint groups were performed by two-way analysis of variance (ANOVA) with a post hoc Tukey test. Repeatedmeasures one-way ANOVA with a post hoc Dunnett's test were used for statistical comparison between pre- and postneedle insertion values. Mean values between groups were compared using the Kruskal-Wallis $\mathrm{H}$ test. The level of statistical significance was set at $P<0.05$. Individual variations were analyzed as percent changes.

\section{Results}

3.1. Participants. One participant assigned to the LR3 group was excluded from the analysis because of arrhythmia. There were no significant differences among the clinical profiles of the 3 groups (Table 1 ).

3.2. Data Summary. Table 2 summarizes the hemodynamic measurements performed before and after needle insertion in the ST36, LR3, and non-acupoint groups. Stimulation at ST36 elicited a decrease in BFV in the BA during needle stimulation compared with that before needle insertion. The basal values of BFV in the BA were not significantly different among the 3 groups. Similarly, no significant differences were observed in any parameters measured at rest before needle insertion.

3.3. Blood Pressure. No significant differences were observed among the 3 groups or with respect to the percent change 


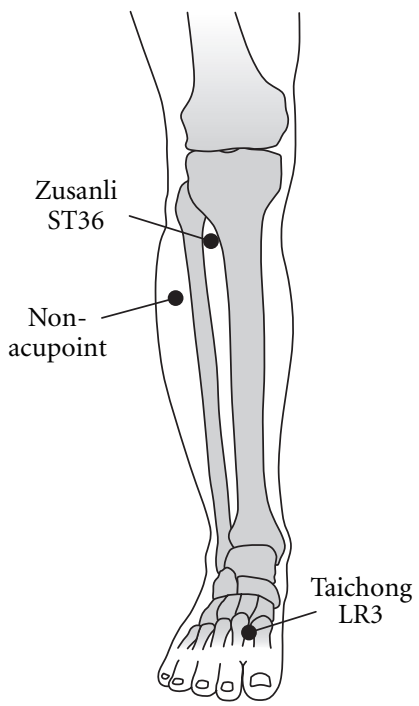

FIGURE 3: Needle positions of ST36, LR3, and the non-acupoint.

TABLE 1: Clinical profiles by group.

\begin{tabular}{lccc}
\hline \multirow{2}{*}{ Profile } & \multicolumn{3}{c}{ Stimulation points } \\
& ST36 & LR3 & Non-acupoint \\
\hline Person & 25 & 24 & 25 \\
Sex (men : women) & $15: 10$ & $14: 10$ & $15: 10$ \\
Age (year) & $31.0 \pm 9.7$ & $34.6 \pm 8.4$ & $33.1 \pm 8.3$ \\
Height $(\mathrm{cm})$ & $166.9 \pm 9.2$ & $167.7 \pm 8.9$ & $165.5 \pm 8.1$ \\
Weight $(\mathrm{kg})$ & $64.0 \pm 12.1$ & $61.7 \pm 10.9$ & $60.3 \pm 9.5$ \\
$\begin{array}{l}\text { Body surface } \\
\text { area }\left(\mathrm{m}^{2}\right)\end{array}$ & $1.71 \pm 0.19$ & $1.70 \pm 0.19$ & $1.66 \pm 0.15$ \\
\hline
\end{tabular}

in systolic or diastolic blood pressure in each test before and after needle insertion.

3.4. Heart Rate. No significant differences were observed among the 3 groups with respect to the percent change in heart rate in each test. Regarding the percent change in heart rate, stimulation at LR3 elicited a significant decrease in heart rate measured during needle stimulation compared to that before needle insertion. The heart rate tended to decrease during needle stimulation.

3.5. Cardiac Index. No significant differences were observed among the 3 groups with respect to the percent change in the $\mathrm{CI}$ in each test. Regarding the percent change in the CI, the stimulation at the non-acupoint elicited a significant increase in the CI in the BA 180 seconds after needle stimulation compared to that before needle insertion.

3.6. Systemic Vascular Resistance Index. No significant differences were observed among the 3 groups with respect to the percent change in SVRI in each test (Figure 4). Regarding the percent change in SVRI, the stimulation at LR3 and the nonacupoint elicited a significant decrease in SVRI in the BA
180 seconds after needle stimulation compared to that before needle insertion.

3.7. Blood Flow Volume of the Brachial Artery. Figure 5 shows the percent change in BFV in the BA in each test. The percent change in BFV in the BA was significantly different between the LR3 group and the other 2 groups. The stimulation at LR3 elicited a significant increase in BFV in the BA 60 and 180 seconds after needle stimulation compared to that before needle insertion. Meanwhile, the stimulation at ST36 elicited a significant decrease in BFV in the BA during and 60 and 180 seconds after needle stimulation compared to that before needle insertion. Furthermore, stimulation at the non-acupoint elicited a significant decrease in BFV in the BA during and 60 seconds after needle stimulation compared to that before needle insertion.

\section{Discussion}

To our knowledge, this is the first study comparing the differences in BFV in the BA induced by needle stimulation at 3 acupoints as assessed by ultrasonography. The results show that BFV in the BA increased significantly after needle stimulation at LR3, whereas it decreased significantly after needle stimulation at ST36 and the non-acupoint. Furthermore, after needle stimulation at the non-acupoint, the CI increased significantly while SVRI decreased significantly. These results indicate that the physiological effects on the BFV in the BA triggered by needle stimulation vary depending on the acupoint stimulated. Needle stimulation at the lower limbs is known to elicit systemic visceral responses via supraspinal reflexes [30-32]. However, visceral responses vary, as observed in the present study.

Stimulation at ST36 and the non-acupoint elicited significant decreases in BFV in the BA during needle stimulation compared to that before needle insertion. The physiological mechanism involved in the decrease in BFV in the BA 
TABLE 2: Summary of measured hemodynamic parameters.

\begin{tabular}{|c|c|c|c|c|c|}
\hline Parameter/test condition & $\begin{array}{c}\text { Before } \\
\text { needle ins }\end{array}$ & $\begin{array}{c}\text { During } \\
\text { needle stim }\end{array}$ & $\begin{array}{c}30 \mathrm{~s} \text { after } \\
\text { needle stim }\end{array}$ & $\begin{array}{c}60 \mathrm{~s} \text { after } \\
\text { needle stim }\end{array}$ & $\begin{array}{c}180 \mathrm{~s} \text { after } \\
\text { needle stim }\end{array}$ \\
\hline \multicolumn{6}{|c|}{ Systolic blood pressure (mm Hg) } \\
\hline ST36 & $116.1 \pm 11.0$ & & & & $116.2 \pm 10.2$ \\
\hline LR3 & $114.7 \pm 12.7$ & & & & $112.1 \pm 12.3$ \\
\hline Non-acupoint & $117.5 \pm 15.5$ & & & & $116.0 \pm 14.0$ \\
\hline \multicolumn{6}{|c|}{ Diastolic blood pressure (mm Hg) } \\
\hline ST36 & $69.1 \pm 8.0$ & & & & $69.0 \pm 8.6$ \\
\hline LR3 & $68.2 \pm 9.1$ & & & & $66.1 \pm 9.0$ \\
\hline Non-acupoint & $70.4 \pm 12.2$ & & & & $70.0 \pm 11.0$ \\
\hline \multicolumn{6}{|l|}{ Heart rate (beats/min) } \\
\hline ST36 & $66.2 \pm 10.2$ & $64.1 \pm 10.7$ & $67.0 \pm 10.7$ & $66.4 \pm 10.5$ & $68.0 \pm 11.7$ \\
\hline LR3 & $63.4 \pm 9.0$ & $60.4 \pm 7.8$ & $63.0 \pm 9.1$ & $63.4 \pm 9.1$ & $63.6 \pm 9.1$ \\
\hline Non-acupoint & $65.0 \pm 9.5$ & $62.6 \pm 10.5$ & $64.5 \pm 9.4$ & $64.8 \pm 9.6$ & $64.8 \pm 10.4$ \\
\hline \multicolumn{6}{|c|}{ Cardiac index $\left(\mathrm{L} \cdot \mathrm{min}^{-1} \cdot \mathrm{m}^{-2}\right)$} \\
\hline ST36 & $3.0 \pm 0.7$ & $3.0 \pm 0.6$ & $3.0 \pm 0.6$ & $3.0 \pm 0.7$ & $3.0 \pm 0.7$ \\
\hline LR3 & $2.7 \pm 0.4$ & $2.7 \pm 0.4$ & $2.7 \pm 0.5$ & $2.7 \pm 0.5$ & $2.7 \pm 0.5$ \\
\hline Non-acupoint & $2.7 \pm 0.4$ & $2.7 \pm 0.4$ & $2.7 \pm 0.4$ & $2.8 \pm 0.4$ & $2.8 \pm 0.4$ \\
\hline \multicolumn{6}{|c|}{$\begin{array}{l}\text { Systemic vascular resistance index } \\
\left(\text { dyne } \mathrm{sec} / \mathrm{cm}^{5} \mathrm{~m}^{2} \text { ) }\right.\end{array}$} \\
\hline ST36 & $2390 \pm 513$ & & & & $2347 \pm 556$ \\
\hline LR3 & $2507 \pm 429$ & & & & $2414 \pm 431$ \\
\hline Non-acupoint & $2602 \pm 654$ & & & & $2522 \pm 586$ \\
\hline \multicolumn{6}{|c|}{$\begin{array}{l}\text { Blood flow volume in brachial artery } \\
\left(\mathrm{mL} \cdot \mathrm{min}^{-1} \cdot \mathrm{m}^{-2}\right)\end{array}$} \\
\hline ST36 & $55.4 \pm 29.3^{* *}$ & $33.4 \pm 13.1^{* *}$ & $48.0 \pm 21.5$ & $44.0 \pm 20.8$ & $45.5 \pm 25.4$ \\
\hline LR3 & $38.9 \pm 25.9$ & $29.8 \pm 20.6$ & $41.8 \pm 25.1$ & $47.7 \pm 30.7$ & $54.8 \pm 32.6$ \\
\hline Non-acupoint & $59.0 \pm 52.4$ & $37.7 \pm 31.0$ & $57.8 \pm 50.6$ & $48.0 \pm 40.5$ & $53.3 \pm 40.7$ \\
\hline
\end{tabular}

The values represent the mean $\pm \mathrm{SD}$. ${ }^{* *}$ Significant difference $(P<0.01)$ in the blood flow volume in the brachial artery between before needle insertion (ins) and during needle stimulation (stim) at ST36.

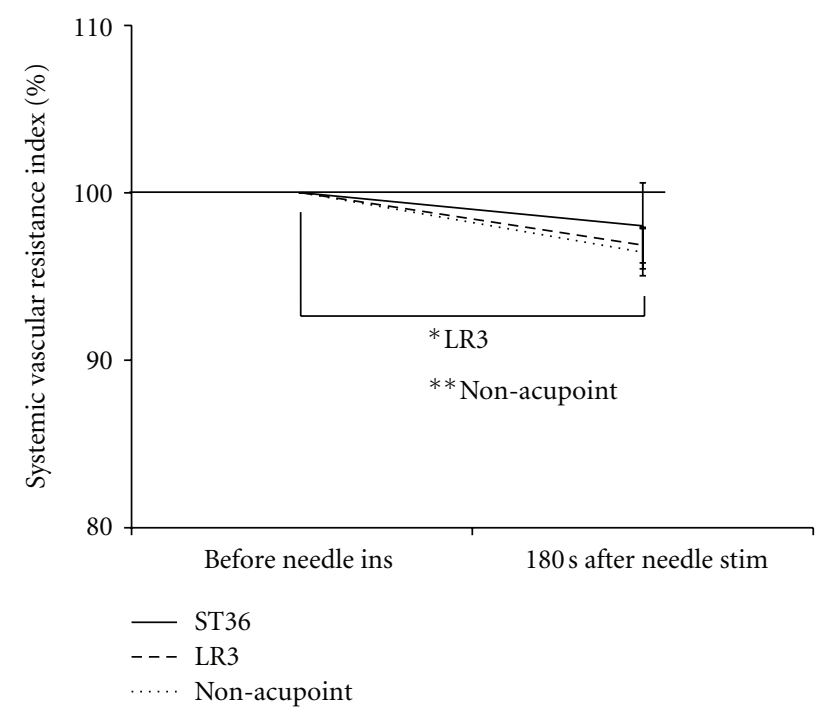

FIGURE 4: Percent change in the systemic vascular resistance index before needle insertion (ins) and at stimulation (stim) at ST36, LR3, and the non-acupoint. Values represent means and standard errors (SEM). ${ }^{*} * *$ Significantly different $(P<0.05, P<0.01$, resp. $)$ relative to the resting condition in each test setup. 


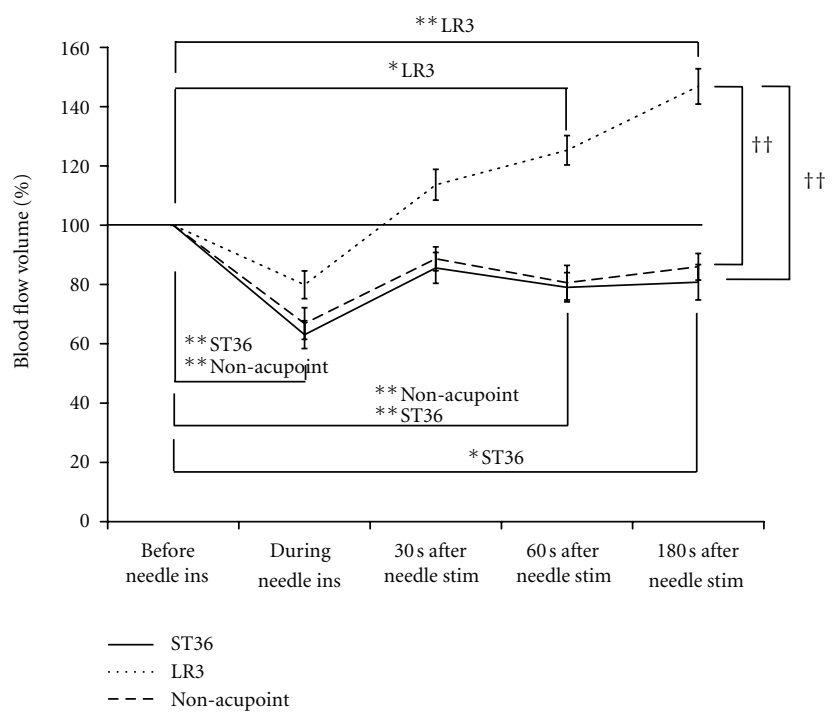

FIGURE 5: Percent change in blood flow volume in the brachial artery before needle insertion (ins) and during and after needle stimulation (stim) at ST36, LR3, and the non-acupoint. The values represent means and corresponding standard errors (SEM). *,** Significant difference $(P<0.05$ and $P<0.01$, resp. $)$ relative to the blood flow volume before needle insertion at LR3, ST36, or the non-acupoint. ${ }^{\dagger \dagger}$ Significant difference $(P<0.01)$ between the LR3 and ST36/non-acupoint groups.

induced by the stimulations applied to the skin is a peripheral vascular resistance caused by an instantaneous increase in sympathetic tone [33]. During needle stimulation, heart rate tended to decrease without a change in the CI. Previous studies report that the mechanism of the acupuncture induced decrease in heart rate is based on somatoautonomic reflexes, which occur via the cardiac sympathetic nerves [34-36]. These mechanisms are involved in the supraspinal reflex.

Stimulation at LR3 elicited a significant increase in BFV in the BA 60 and 180 seconds after needle stimulation compared to that before needle insertion. In addition, for LR3 stimulation, a systemic reaction was elicited such that the SVRI decreased significantly 180 seconds after needle stimulation without any changes in blood pressure, heart rate, or the $\mathrm{CI}$ compared to those before needle insertion. This reaction suggests that the increase of BFV in the BA after stimulation at LR3 depends on the decrease of SVRI. A previous report suggests that the changes in BFV in the BA caused by needle stimulation at LR3 depend on the decrease of the resistive index of the peripheral artery after needle stimulation [13]. This report supports the relationship between the increase of BFV in the BA and the decrease of SVRI observed in the present study. A previous study found that adrenal sympathetic nerve activity and catecholamine secretion rate increase as a result of stimulating the hind limb of rats [30,37-39]. In addition, vasodilation is reported to be elicited via adrenalin beta-2 receptor activation [40]. However, in the present study, the BFV of the BA decreased after needle stimulation at the non-acupoint with the decrease of SVRI. This result may suggest that BFV increased in other organs but not in the BA and that the shift of BFV to other organs is induced by needle stimulation. Similar speculation can be applied to the reaction with ST36 stimulation. However, further investigations are required to clarify these reactions.
The physiological reactions mentioned earlier may support the idea that stimulations at ST36, LR3, and the nonacupoint have different impacts on the autonomic nervous system, which regulates BFV in the BA. A recent report also suggested that opposite cardiovascular effects were shown in the response to stimulation of LR3 and ST36 in rats [41]. We can deduce that the various reactions mediated by the autonomic nervous system depend on the characteristics of a specific stimulation, such as the site, intensity, and duration. The 3 points used in this study (ST36, LR3, and the non-acupoint) are located along the boundary of the same dermatomes, and their innervations are complex [42]. Therefore, each point likely has different sensitivities to stimulation. Among other factors, differences in signal transmission via afferent and efferent fibers depend on anatomical structures such as the dermatomes and myotomes as well as the innervation and thickness of the muscle and skin at the stimulation points.

The present study was a human observational study. Measuring sympathetic and parasympathetic activities in human subjects is invasive and difficult. Therefore, we observed the effects of stimulation at 2 acupoints and 1 nonacupoint on BFV and speculated on the mechanism using previous experimental studies as a basis. In a previous study with 25 participants per group, we reported the changes in BFV in the BA measured by ultrasonography when acupuncture was performed at a single acupoint $[12,13]$. According to these results, we enrolled 25 participants in each of the 3 groups in the present study. The use of ultrasound methods to assess FMD has a good reproducibility [43-45]. However, a previous study evaluating the reproducibility of FMD indicates that 62 subjects per group are required to detect a treatment difference of $2 \mathrm{FMD} \%$ with a probability of 0.05 and a power of 0.80 in a parallel design comparing intergroup changes [46]. Therefore, the small sample size 
of each group may be a limitation of the present study. The findings of this study suggest that acupuncture induces endothelium-dependent FMD, providing an interesting pathophysiological basis for the explanation of observed blood flow changes.

\section{Conclusions}

The present study revealed that BFV in the BA increased after needle stimulation at LR3 without changes in the CI. Needle stimulation at ST36 and the non-acupoint affected the hemodynamics of the BA. These physiological effects may be dependent on acupoint location.

\section{Conflict of Interests}

No conflict of interests exists.

\section{Acknowledgments}

The authors would like to thank all of the participants. M. Watanabe planned the study, recruited participants, performed the experiments, performed data analysis, and wrote the paper. S. Takayama planned the study, provided advice on the experiments, and provided advice on the paper. A. Hirano performed the experiments. T. Seki planned the study and provided advice on the paper. N. Yaegashi was the authority for the study planning as well as execution, and assisted paper writing. This work was supported by Health and Labour Science Research Grants for Clinical Research from the Japanese Ministry of Health, Labour, and Welfare.

\section{References}

[1] M. Giovanni, The Foundations of Chinese Medicine: A Comprehensive Text for Acupuncturists and Herbalists, Churchill Livingstone, Edinburgh, UK, 1989.

[2] S. P. C. Ngai, A. Y. M. Jones, and E. K. W. Cheng, "Lung meridian acupuncture point skin impedance in asthma and description of a mathematical relationship with $\mathrm{FEV}_{1}$," Respiratory Physiology and Neurobiology, vol. 179, no. 2-3, pp. 187191, 2011.

[3] J. A. Sutherland, "Meridian therapy: current research and implications for critical care," AACN Clinical Issues, vol. 11, no. 1, pp. 97-104, 2000.

[4] G. Liu, A. Hyodo, Q. Cao et al., Fundamentals of Acupuncture \& Moxibustion, Huaxia Publishing House, Beijing, China, 2006.

[5] E. Haker, H. Egekvist, and P. Bjerring, "Effect of sensory stimulation (acupuncture) on sympathetic and parasympathetic activities in healthy subjects," Journal of the Autonomic Nervous System, vol. 79, no. 1, pp. 52-59, 2000.

[6] Y. Syuu, H. Matsubara, T. Kiyooka et al., "Cardiovascular beneficial effects of electroacupuncture at Neiguan (PC-6) acupoint in anesthetized open-chest dog," Japanese Journal of Physiology, vol. 51, no. 2, pp. 231-238, 2001.

[7] W. K. Wang, T. Lin Hsu, H. C. Chang, and Y. Y. Lin Wang, "Effect of acupuncture at Hsien-Ku (St-43) on the pulse spectrum and a discussion of the evidence for the frequency structure of Chinese medicine," American Journal of Chinese Medicine, vol. 28, no. 1, pp. 41-55, 2000.

[8] Y. Kurono, M. Minagawa, T. Ishigami, A. Yamada, T. Kakamu, and J. Hayano, "Acupuncture to Danzhong but not to Zhongting increases the cardiac vagal component of heart rate variability," Autonomic Neuroscience, vol. 161, no. 1-2, pp. 116120, 2011.

[9] L. Tough, "Lack of effect of acupuncture on electromyographic (EMG) activity - a randomised controlled trial in healthy volunteers," Acupuncture in Medicine, vol. 24, no. 2, pp. 55-60, 2006.

[10] R. Appiah, S. Hiller, L. Caspary, K. Alexander, and A. Creutzig, "Treatment of primary Raynaud's syndrome with traditional Chinese acupuncture," Journal of Internal Medicine, vol. 241, no. 2, pp. 119-124, 1997.

[11] T. C. Kuo, C. W. Lin, and F. M. Ho, "The soreness and numbness effect of acupuncture on skin blood flow," American Journal of Chinese Medicine, vol. 32, no. 1, pp. 117-129, 2004.

[12] S. Takayama, T. Seki, N. Sugita et al., "Radial artery hemodynamic changes related to acupuncture," Explore, vol. 6, no. 2, pp. 100-105, 2010.

[13] S. Takayama, T. Seki, M. Watanabe et al., "Brief effect of acupuncture on the peripheral arterial system of the upper limb and systemic hemodynamics in humans," Journal of Alternative and Complementary Medicine, vol. 16, no. 7, pp. 707-713, 2010.

[14] M. Watanabe, S. Takayama, and T. Seki, "Haemodynamic changes in the superior mesenteric artery induced by acupuncture stimulation on the lower limbs," Evidence-Based Complementary and Alternative Medicine, vol. 2012, Article ID 908546, 9 pages, 2012.

[15] C. L. Springfield, F. Sebat, D. Johnson, S. Lengle, and C. Sebat, "Utility of impedance cardiography to determine cardiac vs. noncardiac cause of dyspnea in the emergency department," Congestive Heart Failure, vol. 10, no. 2, pp. 14-16, 2004.

[16] A. C. Perrino, A. Lippman, C. Ariyan, T. Z. O’Connor, and M. Luther, "Intraoperative cardiac output monitoring: comparison of impedance cardiography and thermodilution," Journal of Cardiothoracic and Vascular Anesthesia, vol. 8, no. 1, pp. 24-29, 1994.

[17] N. M. Albert, M. D. Hail, J. Li, and J. B. Young, "Equivalence of the bioimpedance and thermodilution methods in measuring cardiac output in hospitalized patients with advanced, decompensated chronic heart failure," American Journal of Critical Care, vol. 13, no. 6, pp. 469-479, 2004.

[18] J. L. Fellahi, V. Caille, C. Charron, P. H. Deschamps-Berger, and A. Vieillard-Baron, "Noninvasive assessment of cardiac index in healthy volunteers: a comparison between thoracic impedance cardiography and doppler echocardiography," Anesthesia and Analgesia, vol. 108, no. 5, pp. 1553-1559, 2009.

[19] J. Soga, K. Nishioka, S. Nakamura et al., "Measurement of flow-mediated vasodilation of the brachial artery-a comparison of measurements in the seated and supine positions," Circulation Journal, vol. 71, no. 5, pp. 736-740, 2007.

[20] U. Gembruch, "Assessment of the fetal circulatory state in uteroplacental insufficiency by Doppler ultrasound: which vessels are the most practicable?" Ultrasound in Obstetrics \& Gynecology, vol. 8, no. 2, pp. 77-81, 1996.

[21] P. N. Burns and C. C. Jaffe, "Quamtitative flow measurements with Doppler ultrasound: techniques, accuracy, and limitations," Radiologic Clinics of North America, vol. 23, no. 4, pp. 641-657, 1985. 
[22] K. J. W. Taylor and S. Holland, "Doppler US_part I. Basic principles, instrumentation, and pitfalls," Radiology, vol. 174, no. 2, pp. 297-307, 1990.

[23] R. W. Gill, "Measurement of blood flow by ultrasound: accuracy and sources of error," Ultrasound in Medicine and Biology, vol. 11, no. 4, pp. 625-641, 1985.

[24] J. Deanfield, A. Donald, C. Ferri et al., "Endothelial function and dysfunction-part I: methodological issues for assessment in the different vascular beds: a statement by the working group on endothelin and endothelial factors of the European society of hypertension," Journal of Hypertension, vol. 23, no. 1, pp. 7-17, 2005.

[25] M. C. Corretti, T. J. Anderson, E. J. Benjamin et al., "Guidelines for the ultrasound assessment of endothelial-dependent flow-mediated vasodilation of the brachial artery: a report of the international brachial artery reactivity task force," Journal of the American College of Cardiology, vol. 39, no. 2, pp. 257265, 2002.

[26] Y. Nimura, H. Matsuo, and T. Hayashi, "Studies on arterial flow patterns-instantaneous velocity spectrums and their phasic changes - with directional ultrasonic Doppler technique," British Heart Journal, vol. 36, no. 9, pp. 899-907, 1974.

[27] C. W. Hsieh, C. W. Mao, M. S. Young, T. L. Yeh, and S. J. Yeh, "Respiratory effect on the pulse spectrum," Journal of Medical Engineering and Technology, vol. 27, no. 2, pp. 77-84, 2003.

[28] D. Korpas and J. Halek, "Pulse wave variability within two short-term measurements," Biomedical Papers of the Medical Faculty of the University Palacký, Olomouc, Czechoslovakia, vol. 150, no. 2, pp. 339-344, 2006.

[29] C. H. Chen, Acupuncture: A Comprehensive Text, Eastland Press, Seattle, Wash, USA, 1981.

[30] A. Sato, Y. Sato, A. Suzuki, and S. Uchida, "Reflex modulation of catecholamine secretion and adrenal sympathetic nerve activity by acupuncture-like stimulation in anesthetized rat," Japanese Journal of Physiology, vol. 46, no. 5, pp. 411-421, 1996.

[31] S. Uchida and H. Hotta, "Acupuncture affects regional blood flow in various organs," Evidence-Based Complementary and Alternative Medicine, vol. 5, no. 2, pp. 145-151, 2008.

[32] E. Noguchi, "Mechanism of reflex regulation of the gastroduodenal function by acupuncture," Evidence-Based Complementary and Alternative Medicine, vol. 5, no. 3, pp. 251-256, 2008.

[33] B. Johansson, "Circulatory responses to stimulation of somatic afferents with special reference to depressor effects from muscle nerves," Acta Physiologica Scandinavica, vol. 198, pp. 1-91, 1962.

[34] S. Uchida, M. Shimura, H. Ohsawa, and A. Suzuki, "Neural mechanism of bradycardiac responses elicited by acupuncture-like stimulation to a hind limb in anesthetized rats," Journal of Physiological Sciences, vol. 57, no. 6, pp. 377-382, 2007.

[35] H. Ohsawa, K. Okada, K. Nishijo, and Y. Sato, "Neural mechanism of depressor responses of arterial pressure elicited by acupuncture-like stimulation to a hindlimb in anesthetized rats," Journal of the Autonomic Nervous System, vol. 51, no. 1, pp. 27-35, 1995.

[36] K. Nishijo, H. Mori, K. Yosikawa, and K. Yazawa, "Decreased heart rate by acupuncture stimulation in humans via facilitation of cardiac vagal activity and suppression of cardiac sympathetic nerve," Neuroscience Letters, vol. 227, no. 3, pp. 165-168, 1997.

[37] J. Li, J. Li, Z. Chen et al., "The influence of PC6 on cardiovascular disorders: a review of central neural mechanisms," Acupuncture in Medicine, vol. 30, no. 1, pp. 47-50, 2012.
[38] E. Stener-Victorin, S. Fujisawa, and M. Kurosawa, "Ovarian blood flow responses to electroacupuncture stimulation depend on estrous cycle and on site and frequency of stimulation in anesthetized rats," Journal of Applied Physiology, vol. 101, no. 1, pp. 84-91, 2006.

[39] S. Stock and K. Uvnas-Moberg, "Increased plasma levels of oxytoxin in response to afferent electrical stimulation of the sciatic and vagal nerves and in response to touch and pinch in anaesthetized rats," Acta Physiologica Scandinavica, vol. 132, no. 1, pp. 29-34, 1988.

[40] D. Robertson, Primer on the Autonomic Nervous System, Elsevier, 3rd edition, 2012.

[41] T. Friedemann, X. Shen, J. Bereiter-Hahn, and W. Schwarz, "Regulation of the cardiovascular function by $\mathrm{CO}_{2}$ laser stimulation in anesthetized rats," Lasers in Medical Science, vol. 27, pp. 469-477, 2012.

[42] S. Standring, Gray's Anatomy: The Anatomical Basis of Clinical Practice, Churchill Livingstone Elsevier, UK, 40th edition, 2008.

[43] S. L. Magda, A. O. Ciobanu, and M. Florescu, "Comparative reproducibility of the noninvasive ultrasound methods for the assessment of vascular function," Heart Vessels. In press.

[44] A. E. Donald, J. P. Halcox, M. Charakida et al., "Methodological approaches to optimize reproducibility and power in clinical studies of flow-mediated dilation," Journal of the American College of Cardiology, vol. 51, no. 20, pp. 1959-1964, 2008.

[45] L. Ghiadoni, F. Faita, M. Salvetti et al., "Assessment of flowmediated dilation reproducibility: a nationwide multicenter study," Journal of Hypertension, vol. 30, no. 7, pp. 1399-1405, 2012.

[46] N. M. De Roos, M. L. Bots, E. G. Schouten, and M. B. Katan, "Within-subject variability of flow-mediated vasodilation of the brachial artery in healthy men and women: implications for experimental studies," Ultrasound in Medicine and Biology, vol. 29, no. 3, pp. 401-406, 2003. 


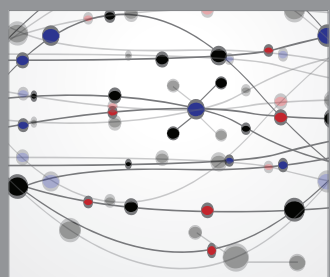

The Scientific World Journal
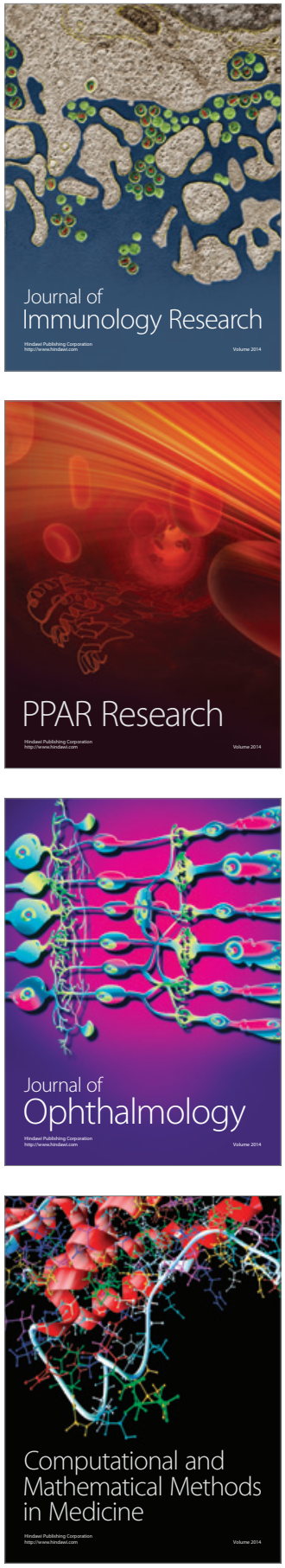

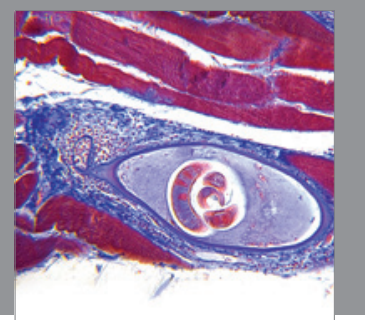

Gastroenterology

Research and Practice
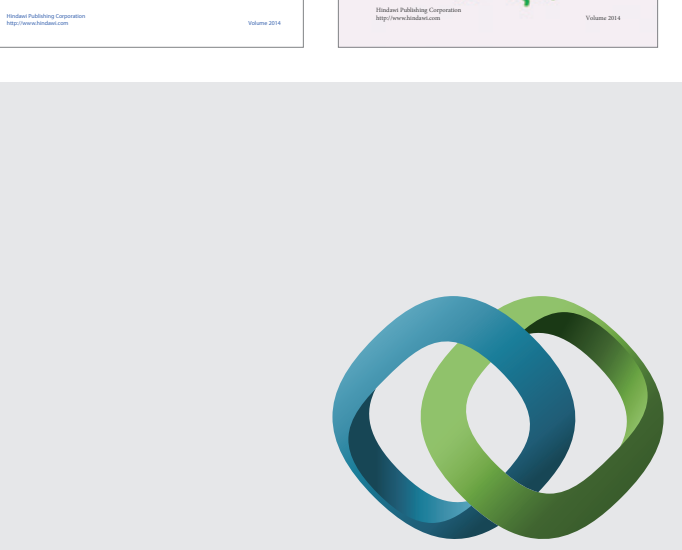

\section{Hindawi}

Submit your manuscripts at

http://www.hindawi.com
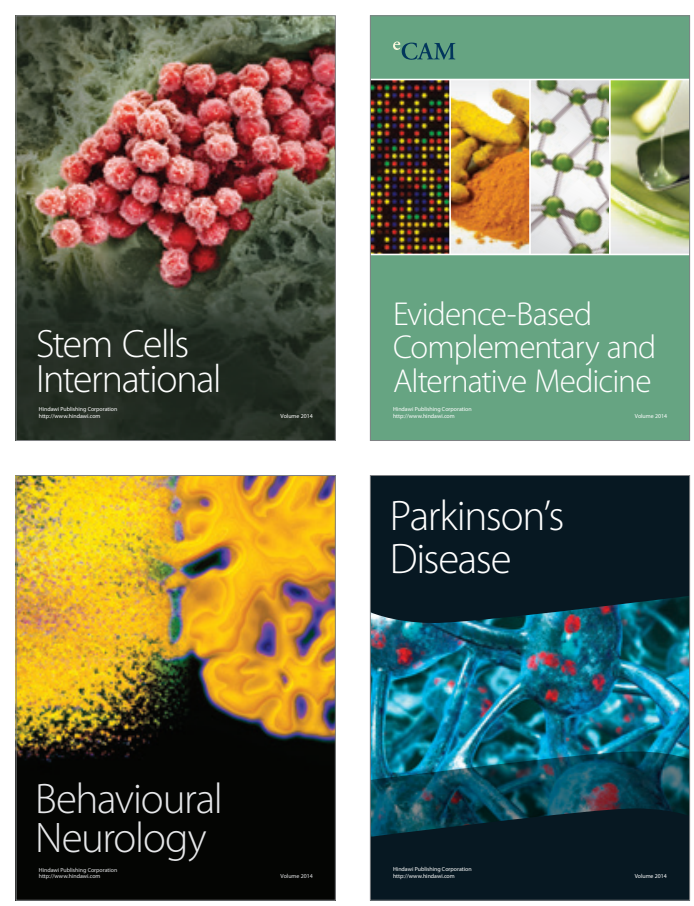

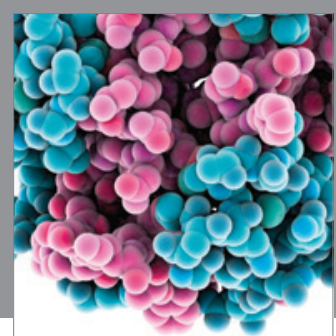

Journal of
Diabetes Research

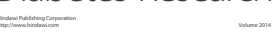

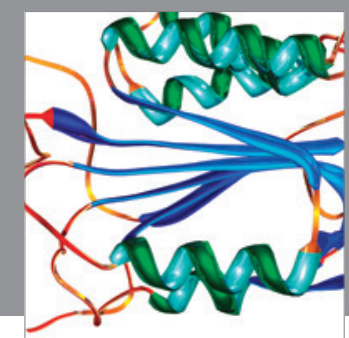

Disease Markers
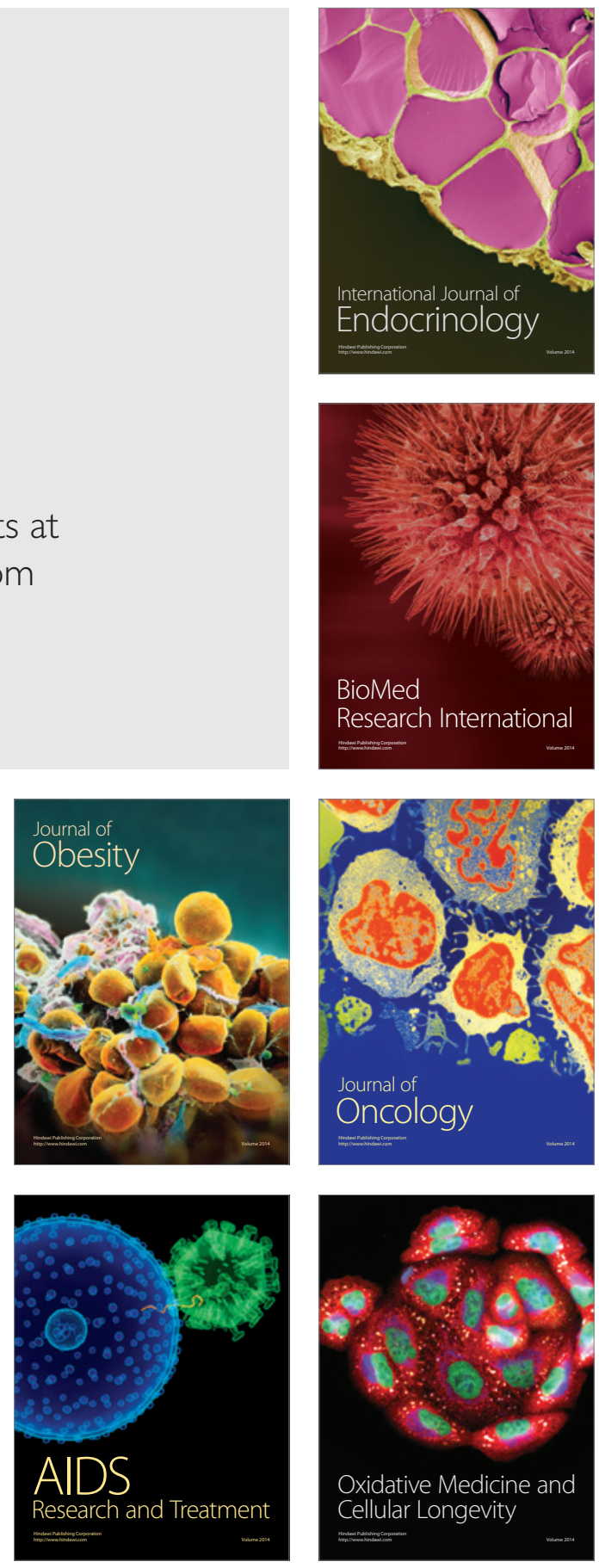